\title{
An Adaptive Human Pilot Model for Adaptively Controlled Systems
}

\author{
Abdullah Habboush ${ }^{\circledR}$ and Yildiray Yildiz ${ }^{\circledR}$, Senior Member, IEEE
}

\begin{abstract}
Despite their success in handling uncertain dynamical systems that are prone to failure, adaptive controllers are observed to have unfavorable interactions with human pilots in certain applications. To alleviate this problem, we need to evaluate the safety and performance of adaptive controllers in the simulation environment using realistic pilot models before conducting flight tests. While many useful human pilot models exist in the literature, models that are adequate for the prediction of adaptive humanadaptive controller interactions are yet to be available. In this letter, we fill this gap by proposing an adaptive human pilot model suited for the prediction of human behavior in the loop with an adaptive controller. The model can serve as a valuable tool guiding the design of adaptive controllers so as to ensure smooth pilot-controller interactions.
\end{abstract}

Index Terms-Adaptive control, adaptive systems, human-in-the-loop control.

\section{INTRODUCTION}

$\mathbf{I}$ RRESPECTIVE of the numerous advances in the field of control theory, the human operator is still irreplaceable in critical tasks where uncertainty in the controlled elements and unexpected external influences can lead to catastrophic events. And with a long way ahead before reaching highly intelligent controllers that can imitate the versatility and the adaptability of human operators in controlling complex systems, several mathematical human pilot models are developed aiming to enhance the aviation safety and handling quality measures by providing valuable guidance to the control system designer.

One of the most important pilot models is the crossover model proposed by McRuer [1], which is derived based on experimental observations that a human operator controls a system in such a way that results in a well-designed linear feedback system. The crossover model is followed by many extensions forming a large pool a designer can refer to for predicting the human behavior in the loop with a wide range of time-invariant controlled elements [2], [3]. But, when it comes

Manuscript received September 14, 2021; revised November 16, 2021; accepted December 1, 2021. Date of publication December 17, 2021; date of current version December 29, 2021. This work was supported by the Scientific and Technological Research Council of Turkey under Grant 118E937. Recommended by Senior Editor M. Guay. (Corresponding author: Abdullah Habboush.)

The authors are with the Department of Mechanical Engineering, Bilkent University, 06800 Ankara, Turkey (e-mail: a.habboush@ bilkent.edu.tr; yyildiz@ bilkent.edu.tr).

Digital Object Identifier 10.1109/LCSYS.2021.3136460 to modeling systems that are prone to failures, damage, and sudden parametric changes, these fixed pilot models fail to resemble how a human is found to respond adaptively in such critical situations. This shortcoming led to the development of a few adaptive human pilot models.

A prominent adaptive human pilot model is the one proposed in [4] and [5], where the adaptation laws are based on expert knowledge, aiming to make the adaptive pilot model follow the dictates of the crossover model. Inspired by this idea, a promising experimentally-validated adaptive human pilot model is recently developed in [6] and [7], by resorting to model reference adaptive control (MRAC) techniques which allows a rigorous stability analysis using the Lyapunov-Krasovskii stability criteria. These studies are conducted assuming the adaptive human pilot is operating on a linear controller-plant combination. Although there exist studies such as [8], [9], where an adaptive controller is in the loop, the pilot model used is not adaptive.

Developing adaptive pilot models that are suitable for adaptively controlled systems is important because owing to their special nonlinear characteristics, unfavorable interactions of human pilots with the adaptive control systems are observed in several flight tests [10].

The field of flight control reconfiguration emerged to address the issue of actuator failures by replacing redundant physical hardware with control algorithms that utilize the remaining control surfaces to compensate for loss of actuator effectiveness [11]. The consideration of such an uncertainty in the early design stage has become a standard approach in adaptive controller design [8], [12]-[15], and in adaptive control allocation methods [9], [16], [17], after its importance is highlighted in early studies such as [18], [19].

In this letter, we propose an adaptive human pilot model suited to be used in the loop with an adaptive controller, for the first time in the literature. The model serves as a tool for testing adaptive controllers, which can help the designer manipulate the controller parameters in order to ensure smooth pilot-controller interactions. The development of the model is carried out based on MRAC architecture, with a rigorous Lyapunov stability analysis.

A preliminary version of this letter can be found in [20]. This letter distinguishes itself from [20] by introducing additional actuator uncertainty. The presence of this uncertainty adds to the complexity of the adaptive pilot model, necessitating two additional adaptive parameters, and imposing a 


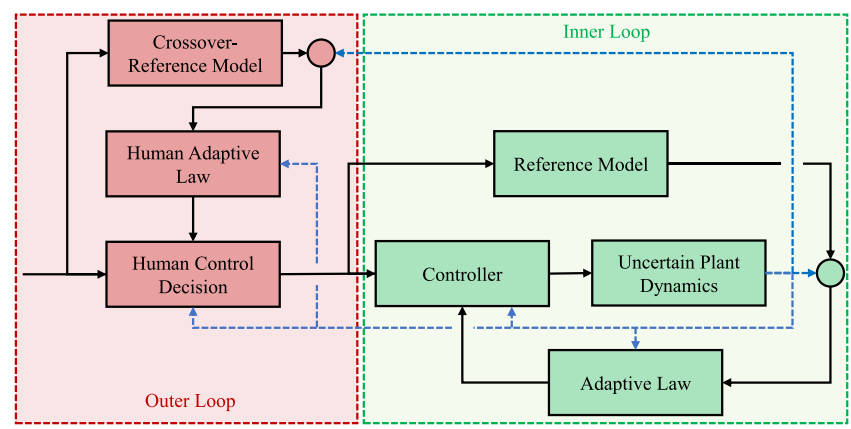

Fig. 1. Block Diagram.

supplemental condition on the adaptive controller. As a result, a time-varying anti-windup design parameter is used to compensate for the additional adaptive parameters, and the design parameter's existence is ensured by guaranteeing the invertibility of the adaptive parameters. In addition, we extend our results to plants with free integrators, where a special treatment is needed to ensure command tracking. The latter allows us to conduct a numerical simulation of a transport aircraft where a human pilot commands all attitude rates using a side-stick aiming to control the vehicle's attitude angles.

The notation used here is standard, where $\mathbb{R}^{p \times q}$ $\left[\mathbb{S}^{p \times q}\right]\left\{\mathbb{D}^{p \times q}\right\}$ denotes the set of real [symmetric real] \{diagonal real\} $\mathrm{p}$ by $\mathrm{q}$ matrices, and $\|\cdot\|$ refers to the euclidean norm for vectors $(q=1)$, and the induced-2 norm for matrices. $\|\cdot\|_{F}$ refers to the Frobenius norm for matrices, $\operatorname{Tr}\{$.$\} refers to the trace operator, and (.)^{T}\left[(.)^{-1}\right]$ denotes the transpose [inverse] operator. Finally, we write $\lambda_{\min }(A)$ for the minimum eigenvalue of the matrix $A$ and we denote the set of positive definite real matrices by $\mathbb{R}_{+}^{p \times p}$.

\section{Problem Statement}

To model the human's adaptive control behavior with an adaptive controller in the loop, we start with a block diagram given in Fig. 1. In the figure, the block diagram is divided into inner and outer loops. The inner-loop consists of an adaptive controller controlling a plant with uncertain dynamics such that the plant states follow those of a reference model by adjusting the control parameters using an adaptive law.

The outer-loop consists of the human controlling the inner-loop such that the plant output follows a reference input. The human is assumed to be well trained, i.e., familiar with the nominal plant-controller dynamics. However, he/she is not aware of the uncertainties in the plant dynamics. This motivates modeling the human as an adaptive outer-loop controller, where an adaptive law is utilized to force the plant states to follow the states of the crossover-reference model.

\section{INNER LOOP}

Consider the following uncertain plant dynamics

$$
\begin{aligned}
& \dot{x}_{p}(t)=A_{p} x_{p}(t)+B_{p} \Lambda u_{p}(t)+B_{p} W_{p}^{T} \sigma_{p}\left(x_{p}(t)\right), \\
& y_{1}(t)=C_{1}^{T} x_{p}(t), y_{2}(t)=C_{2}^{T} x_{p}(t),
\end{aligned}
$$

where $x_{p}(t) \in \mathbb{R}^{n_{p}}$ is the accessible state vector, $u_{p}(t) \in \mathbb{R}^{m}$ is the plant control input, $\sigma_{p}\left(x_{p}(t)\right): \mathbb{R}^{n_{p}} \rightarrow \mathbb{R}^{s}$ is a known possibly non-linear basis function, $W_{p} \in \mathbb{R}^{s \times m}$ is an unknown weight matrix, $\Lambda \in \mathbb{R}_{+}^{m \times m} \cap \mathbb{D}^{m \times m}$ is an unknown control effectiveness matrix with the diagonal elements $\lambda_{i, i} \in(0,1]$, $A_{p} \in \mathbb{R}^{n_{p} \times n_{p}}$ is an unknown system matrix, $B_{p} \in \mathbb{R}^{n_{p} \times m}$ is a known control input matrix, and $C_{1} \in \mathbb{R}^{n_{p} \times m}$ and $C_{2} \in \mathbb{R}^{n_{p} \times r}$ are both known output matrices. The outputs $y_{1}(t) \in \mathbb{R}^{m}$ and $y_{2}(t) \in \mathbb{R}^{r}$ are the outputs of interest for the inner and outer loops, respectively. Furthermore, it is assumed that the pair $\left(A_{p}, B_{p}\right)$ is controllable.

Let the nominal plant dynamics be given as

$$
\dot{x}_{n}(t)=A_{n} x_{n}(t)+B_{p} u_{n}(t)
$$

where $u_{n}(t) \in \mathbb{R}^{m}$ is a nominal controller given as

$$
u_{n}(t)=-L_{x} x_{n}(t)+L_{r} y_{h}(t),
$$

where $y_{h}(t) \in \mathbb{R}^{m}$ is the human command, and $L_{x} \in \mathbb{R}^{m \times n_{p}}$ is such that $A_{r} \triangleq A_{n}-B_{p} L_{x}$ is Hurwitz. It is noted that the human input $y_{h}(t)$ is bounded due to physical manipulator limits. In the design of the outer loop, given in the next section, human input saturation is considered in the stability analysis. Defining $B_{r} \triangleq B_{p} L_{r}$, the reference model is assigned as

$$
\dot{x}_{r}(t)=A_{r} x_{r}(t)+B_{r} y_{h}(t) .
$$

For a constant $y_{h}$, at steady state, it is obtained using (4) that $\dot{x}_{r}(\infty)=0=A_{r} x_{r}(\infty)+B_{r} y_{h}$, and $x_{r}(\infty)=-A_{r}^{-1} B_{p} L_{r} y_{h}$. Hence, once $\lim _{t \rightarrow \infty} x_{p}(t)=x_{r}(t)$, the plant output $y_{1}(t)$, given in (1), takes the form $y_{1}(\infty)=-C_{1}^{T} A_{r}^{-1} B_{p} L_{r} y_{h}$. Selecting

$$
L_{r}=-\left(C_{1}^{T} A_{r}^{-1} B_{p}\right)^{-1},
$$

results in $\lim _{t \rightarrow \infty} y_{1}(t)=y_{h}$.

Considering (1), which can be rewritten as

$$
\dot{x}_{p}(t)=A_{p} x_{p}(t)+B_{p} \Lambda\left(u_{p}(t)+W^{T} \sigma_{p}\left(x_{p}(t)\right)\right),
$$

where $W^{T} \triangleq \Lambda^{-1} W_{p}^{T} \in \mathbb{R}^{m \times s}$ is an unknown parameter, we assume that there exist $K_{x}^{*} \in \mathbb{R}^{m \times n_{p}}$ and $K_{r}^{*} \in \mathbb{R}^{m \times m}$ such that the matching conditions

$$
A_{p}-B_{p} \Lambda K_{x}^{*}=A_{r}, B_{p} \Lambda K_{r}^{*}=B_{r} \triangleq B_{p} L_{r},
$$

are satisfied. The second matching condition implies that $K_{r}^{*}=$ $\Lambda^{-1} L_{r}$. We define the plant control law as

$$
u_{p}(t)=-\hat{K}_{x}(t) x_{p}(t)+\operatorname{diag}(\hat{\lambda}(t)) L_{r} y_{h}(t)-\hat{W}^{T}(t) \sigma_{p}\left(x_{p}(t)\right),
$$

where $\hat{K}_{x}(t) \in \mathbb{R}^{m \times n_{p}}, \hat{\lambda}(t) \in \mathbb{R}^{m}$ and $\hat{W}(t) \in \mathbb{R}^{s \times m}$ are adjustable parameters serving as estimates for the ideal values $K_{x}^{*}, \lambda^{*}$ and $W$, respectively. It is noted that $\operatorname{diag}\left(\lambda^{*}\right)=\Lambda^{-1}$ exists since $\Lambda$ is diagonal positive definite.

Substituting (8) into (6), one can rewrite (6) as

$$
\begin{aligned}
\dot{x}_{p}(t)= & A_{r} x_{p}(t)+B_{r} y_{h}(t)+B_{p} \Lambda \operatorname{diag}(\tilde{\lambda}(t)) L_{r} y_{h}(t) \\
& -B_{p} \Lambda\left(\tilde{W}^{T}(t) \sigma_{p}\left(x_{p}(t)\right)+\tilde{K}_{x}(t) x_{p}(t)\right),
\end{aligned}
$$

where $\tilde{K}_{x}(t) \triangleq \hat{K}_{x}(t)-K_{x}^{*}, \tilde{\lambda}(t) \triangleq \hat{\lambda}(t)-\lambda^{*}$ and $\tilde{W}(t) \triangleq$ $\hat{W}(t)-W$ are the adaptive parameters errors. 
Defining $\Theta(t) \triangleq\left[\hat{W}^{T}(t), \hat{K}_{x}(t)\right]^{T} \in \mathbb{R}^{\left(s+n_{p}\right) \times m}$, with the ideal value $\Theta^{*} \triangleq\left[W^{T}, K_{x}^{*}\right]^{T}$, (9) can be rewritten as

$$
\begin{aligned}
\dot{x}_{p}(t)= & A_{r} x_{p}(t)+B_{r} y_{h}(t)+B_{p} \Lambda \operatorname{diag}(\tilde{\lambda}(t)) L_{r} y_{h}(t) \\
& -B_{p} \Lambda \tilde{\Theta}^{T}(t) \sigma\left(x_{p}(t)\right),
\end{aligned}
$$

where $\tilde{\Theta}(t) \triangleq \Theta(t)-\Theta^{*}$ and $\sigma\left(x_{p}(t)\right) \triangleq\left[\sigma_{p}^{T}\left(x_{p}(t)\right), x_{p}^{T}(t)\right]^{T} \in$ $\mathbb{R}^{s+n_{p}}$.

By subtracting (4) from (10), and exploiting the fact that $\Lambda \operatorname{diag}(\tilde{\lambda}(t)) L_{r} y_{h}(t)=\operatorname{diag}\left(L_{r} y_{h}(t)\right) \Lambda \tilde{\lambda}(t)$, we obtain that

$$
\begin{aligned}
\dot{e}_{1}(t)= & A_{r} e_{1}(t)+B_{p} \operatorname{diag}\left(L_{r} y_{h}(t)\right) \Lambda \tilde{\lambda}(t) \\
& -B_{p} \Lambda \tilde{\Theta}^{T}(t) \sigma\left(x_{p}(t)\right),
\end{aligned}
$$

where $e_{1}(t) \triangleq x_{p}(t)-x_{r}(t)$ is the inner-loop tracking error. We define the inner-loop adaptive laws as

$$
\begin{aligned}
\dot{\tilde{\Theta}}(t) & =\dot{\Theta}(t)=\gamma_{\theta} \sigma\left(x_{p}(t)\right) e_{1}(t)^{T} P_{1} B_{p}, \\
\dot{\tilde{\lambda}}(t) & =\dot{\hat{\lambda}}(t)=\gamma_{\lambda} \operatorname{Proj}\left(\hat{\lambda}(t),-\operatorname{diag}\left(L_{r} y_{h}(t)\right) B_{p}^{T} P_{1} e_{1}(t)\right),
\end{aligned}
$$

where Proj(.,.) is the projection operator [12], [16] used to bound the adaptive parameter $\hat{\lambda}(t)$ in a compact set with each element $\hat{\lambda}_{i}(t)$ having positive upper and lower bounds $\hat{\lambda}_{\text {max }_{i}}$ and $\hat{\lambda}_{\text {min }_{i}}$. Furthermore, $\gamma_{\theta}, \gamma_{\lambda} \in \mathbb{R}_{+}$are learning rates, and $P_{1} \in \mathbb{R}_{+}^{n_{p} \times n_{p}} \cap \mathbb{S}^{n_{p} \times n_{p}}$ is the solution of $A_{r}^{T} P_{1}+P_{1} A_{r}=-Q_{1}$, for some $Q_{1} \in \mathbb{R}_{+}^{n_{p} \times n_{p}} \cap \mathbb{S}^{n_{p} \times n_{p}}$.

Lemma 1: Consider the uncertain dynamical system (1), the reference model (4), and the feedback control law given by (8) and (12). The solution $\left(e_{1}(t), \tilde{\Theta}(t), \tilde{\lambda}(t)\right)$ is Lyapunov stable in the large. Furthermore, since the human command $y_{h}(t)$ is bounded, $\lim _{t \rightarrow \infty} e_{1}(t)=0$ and $\dot{\tilde{\Theta}}(t)$ and $\dot{\tilde{\lambda}}(t)$ remain bounded along with all the signals in the inner-loop.

Proof: Consider the Lyapunov function candidate

$$
V_{1}=e_{1}^{T} P_{1} e_{1}+\gamma_{\theta}^{-1} \operatorname{Tr}\left(\left(\tilde{\Theta} \Lambda^{1 / 2}\right)^{T}\left(\tilde{\Theta} \Lambda^{1 / 2}\right)\right\}+\gamma_{\lambda}^{-1} \tilde{\lambda}^{T} \Lambda \tilde{\lambda} .
$$

Differentiating along the trajectories (11) and (12) yields

$$
\begin{aligned}
\dot{V}_{1}= & \dot{e}_{1}^{T} P_{1} e_{1}+e_{1}^{T} P_{1} \dot{e}_{1}+2 \gamma_{\theta}^{-1} \operatorname{Tr}\left\{\tilde{\Theta}^{T} \dot{\tilde{\Theta}} \Lambda\right\}+2 \gamma_{\lambda}^{-1} \tilde{\lambda}^{T} \Lambda \dot{\tilde{\lambda}} \\
= & -e_{1}^{T} Q_{1} e_{1}+2 \gamma_{\theta}^{-1} \operatorname{Tr}\left\{\tilde{\Theta}^{T} \dot{\tilde{\Theta}} \Lambda\right\}+2 \gamma_{\lambda}^{-1} \tilde{\lambda}^{T} \Lambda \dot{\tilde{\lambda}} \\
& -2 \operatorname{Tr}\left\{\tilde{\Theta}^{T} \sigma\left(x_{p}\right) e_{1}^{T} P_{1} B_{p} \Lambda\right\}+2 \tilde{\lambda}^{T} \Lambda \operatorname{diag}\left(L_{r} y_{h}\right) B_{p}^{T} P_{1} e_{1} \\
= & -e_{1}^{T} Q_{1} e_{1}+2 \tilde{\lambda}^{T} \Lambda\left(\operatorname{Proj}\left(\hat{\lambda},-\operatorname{diag}\left(L_{r} y_{h}\right) B_{p}^{T} P_{1} e_{1}\right)\right. \\
& \left.\quad+\operatorname{diag}\left(L_{r} y_{h}\right) B_{p}^{T} P_{1} e_{1}\right) .
\end{aligned}
$$

Using the property $\left(\theta_{i, j}-\theta_{i, j}^{*}\right)\left(\operatorname{Proj}\left(\theta_{i, j}, Y_{i, j}\right)-Y_{i, j}\right) \leq 0$ and the fact that $\Lambda$ is diagonal positive definite, it follows that

$$
\dot{V}_{1} \leq-e_{1}^{T} Q_{1} e_{1} \leq 0
$$

Hence, the solution $\left(e_{1}(t), \tilde{\Theta}(t), \tilde{\lambda}(t)\right)$ is stable in the large.

Equation (4) indicates that the boundedness of $y_{h}(t)$ implies the boundedness of $x_{r}(t)$. The latter coupled with the fact that $e_{1}(t)$ is bounded imply the boundedness of $x_{p}(t)$, which in turn implies that $\left(\dot{e}_{1}(t), \dot{\tilde{\Theta}}(t), \dot{\tilde{\lambda}}(t)\right)$ are bounded. It then follows from Barbalat's lemma that $\lim _{t \rightarrow \infty} e_{1}(t)=0$.

\section{OUTER LOOP}

Using (7), and $\operatorname{diag}\left(\lambda^{*}\right)=\Lambda^{-1}$, (10) can be rewritten as

$$
\begin{aligned}
\dot{x}_{p}(t)= & A_{r} x_{p}(t)+B_{p} \Lambda \operatorname{diag}(\hat{\lambda}(t)) L_{r} y_{h}(t) \\
& -B_{p} \Lambda \tilde{\Theta}^{T}(t) \sigma\left(x_{p}(t)\right) .
\end{aligned}
$$

Since we assume that the human operator is familiar with the nominal dynamics (2) and (3), the only unknowns in (16) for the outer loop are the parameters $\Lambda, \hat{\lambda}(t)$ and $\tilde{\Theta}(t)$.

Consider the integral action

$$
\dot{x}_{c}(t)=y_{2}(t)-r(t)+J(t) \Delta y(t),
$$

where $x_{c}(t) \in \mathbb{R}^{r}$ is the integrator state, $r(t) \in \mathbb{R}^{r}$ is a bounded reference input, and $J(t) \in \mathbb{R}^{r \times m}$ is a control parameter defined below. The term $J(t) \Delta y(t)$, which will be explained later, is included to avoid integrator windup, and it only takes action when the human input is saturated [21], [22]. Aggregating (16) and (17) results in the augmented dynamics

$$
\begin{aligned}
\dot{x}(t)= & A x(t)+B_{m} r(t)-B_{m} J(t) \Delta y(t) \\
& +B H^{T}(t) \sigma\left(x_{p}(t)\right)+B \Lambda_{2}(t) L_{r} y_{h}(t),
\end{aligned}
$$

where $x(t) \triangleq\left[x_{p}^{T}(t), x_{c}^{T}(t)\right]^{T} \in \mathbb{R}^{n}$ is the augmented state vector with $n=n_{p}+r, H^{T}(t) \triangleq-\Lambda \tilde{\Theta}^{T}(t)$ and $\Lambda_{2}(t) \triangleq$ $\Lambda \operatorname{diag}(\hat{\lambda}(t))$ are unknown time-varying parameters, and

$$
\begin{aligned}
A & \triangleq\left[\begin{array}{cc}
A_{r} & 0_{n_{p} \times r} \\
C_{2}^{T} & 0_{r \times r}
\end{array}\right] \in \mathbb{R}^{n \times n}, \\
B & \triangleq\left[\begin{array}{ll}
B_{p}^{T} & 0_{m \times r}
\end{array}\right]^{T} \in \mathbb{R}^{n \times m}, \\
B_{m} & \triangleq\left[\begin{array}{ll}
0_{r \times n_{p}} & -I_{r \times r}
\end{array}\right]^{T} \in \mathbb{R}^{n \times r} .
\end{aligned}
$$

The goal of the human is to control the system such that the plant states follow that of a unity feedback reference model with an open loop crossover model transfer function. We refer to the latter as the crossover-reference model (Fig. 1). Let the crossover-reference model be given as

$$
\dot{x}_{m}(t)=A_{m} x_{m}(t)+B_{m} r(t),
$$

where $x_{m}(t) \in \mathbb{R}^{n}$ is the crossover-reference model state vector, and $A_{m} \in \mathbb{R}^{n \times n}$ is Hurwitz.

In an ideal case where the human input is not saturated, and both $H(t)$ and $\Lambda_{2}(t)$ are known, the following control law achieves the crossover-reference model dynamics

$$
\begin{aligned}
\mathcal{G}^{*}(t) & =-\theta_{x} x(t)-L_{r}^{-1} H^{T}(t) \sigma\left(x_{p}(t)\right), \\
y_{h}^{*}(t) & =L_{r}^{-1} \Lambda_{2}^{-1}(t) L_{r} \mathcal{G}^{*}(t),
\end{aligned}
$$

where we assume that there exists $\theta_{x} \in \mathbb{R}^{m \times n}$ such that $A_{m}=A-B L_{r} \theta_{x}$. We define the human control input by augmenting the baseline PI-control term $-\theta_{x} x(t)$ with adaptive components as

$$
\begin{aligned}
\mathcal{G}(t) & =-\theta_{x} x(t)-L_{r}^{-1} \hat{H}^{T}(t) \sigma\left(x_{p}(t)\right), \\
v(t) & =L_{r}^{-1} \operatorname{diag}\left(\hat{\lambda}_{2}(t)\right) L_{r} \mathcal{G}(t), \\
y_{h_{i}}(t) & = \begin{cases}v_{i}(t), & \text { if }\left|v_{i}(t)\right| \leq y_{o_{i}}, \\
y_{o_{i}} \operatorname{sgn}\left(v_{i}(t)\right), & \text { if }\left|v_{i}(t)\right|>y_{o_{i}},\end{cases}
\end{aligned}
$$

where $\hat{H}(t)$ and $\hat{\lambda}_{2}(t)$ are adjustable parameters serving as estimates for the ideal values $H(t)$ and $\lambda_{2}^{*}(t)$, respectively. It is 
noted that $\operatorname{diag}\left(\lambda_{2}^{*}(t)\right)=\Lambda_{2}^{-1}(t)$ exists for all $t \geq 0$ since $\Lambda_{2}(t)$ is diagonal positive definite at all time instants. Finally, $(22 \mathrm{c})$ is an element-wise saturation function where $y_{o_{i}} \in \mathbb{R}_{+}$is the saturation limit of $y_{h_{i}}(t)$ (the $i^{\text {th }}$ element of $y_{h}(t)$ ).

Remark 1: Such a $\theta_{x}$ exists only if $A_{m}$ is selected such that

$$
A_{m} \triangleq\left[\begin{array}{cc}
A_{r}-B_{r} \phi & -B_{r} \psi \\
C_{2}^{T} & 0_{r \times r}
\end{array}\right] \in \mathbb{R}^{n \times n},
$$

for some $\phi \in \mathbb{R}^{m \times n_{p}}$ and $\psi \in \mathbb{R}^{m \times r}$. Then, $\theta_{x}$ can be assigned as

$$
\theta_{x} \triangleq\left[\begin{array}{ll}
\phi & \psi
\end{array}\right] \in \mathbb{R}^{m \times n} .
$$

Assumption 1: It is assumed that there exists a matrix $M \in$ $\mathbb{R}^{r \times m}$ such that $M \psi \in \mathbb{R}^{r \times r}$ is Hurwitz. This assumption is used in Theorem 1 below.

Substituting (22) into (18), we obtain that

$$
\begin{aligned}
\dot{x}(t)= & A_{m} x(t)+B_{m} r(t)-B_{m} J(t) \Delta y(t)+B \Lambda_{2}(t) L_{r} \Delta y(t) \\
& -B \tilde{H}^{T}(t) \sigma\left(x_{p}(t)\right)+B \Lambda_{2}(t) \operatorname{diag}\left(\tilde{\lambda}_{2}(t)\right) L_{r} \mathcal{G}(t),
\end{aligned}
$$

where $\tilde{H}(t) \triangleq \hat{H}(t)-H(t)$ and $\tilde{\lambda}_{2}(t) \triangleq \hat{\lambda}_{2}(t)-\lambda_{2}^{*}(t)$ are outer-loop adaptive parameters errors, and $\Delta y(t) \triangleq y_{h}(t)-v(t)$ is the control deficiency due to human input saturation.

Subtracting (20) from (25), and exploiting the fact that $\Lambda_{2}(t) \operatorname{diag}\left(\tilde{\lambda}_{2}(t)\right) L_{r} \mathcal{G}(t)=\operatorname{diag}\left(L_{r} \mathcal{G}(t)\right) \Lambda_{2}(t) \tilde{\lambda}_{2}(t)$ results in the outer-loop error dynamics

$$
\begin{aligned}
\dot{e}_{2}(t)= & A_{m} e_{2}(t)-B_{m} J(t) \Delta y(t)+B \Lambda_{2}(t) L_{r} \Delta y(t) \\
& -B \tilde{H}^{T}(t) \sigma\left(x_{p}(t)\right)+B \operatorname{diag}\left(L_{r} \mathcal{G}(t)\right) \Lambda_{2}(t) \tilde{\lambda}_{2}(t),
\end{aligned}
$$

where $e_{2}(t) \triangleq x(t)-x_{m}(t)$ is the outer-loop tracking error.

We generate an auxiliary signal $e_{\Delta}(t)$ as in [13], [23]

$$
\begin{aligned}
\dot{e}_{\Delta}(t) & =A_{m} e_{\Delta}(t)-B_{m} J(t) \Delta y(t)+B \operatorname{diag}\left(\hat{\lambda}_{3}(t)\right) L_{r} \Delta y(t), \\
e_{\Delta}\left(t_{0}\right) & =0
\end{aligned}
$$

where $\hat{\lambda}_{3}(t)$ is an adjustable parameter serving as an estimate for the ideal value $\lambda_{3}^{*}(t)$, and $\operatorname{diag}\left(\lambda_{3}^{*}(t)\right)=\Lambda_{2}(t)$. Defining an augmented error signal $e_{y}(t) \triangleq e_{2}(t)-e_{\Delta}(t)$, and using the fact that $\operatorname{diag}\left(\tilde{\lambda}_{3}(t)\right) L_{r} \Delta y(t)=\operatorname{diag}\left(L_{r} \Delta y(t)\right) \tilde{\lambda}_{3}(t)$ yields

$$
\begin{aligned}
\dot{e}_{y}(t)= & A_{m} e_{y}(t)-B \operatorname{diag}\left(L_{r} \Delta y(t)\right) \tilde{\lambda}_{3}(t) \\
& -B \tilde{H}^{T}(t) \sigma\left(x_{p}(t)\right)+B \operatorname{diag}\left(L_{r} \mathcal{G}(t)\right) \Lambda_{2}(t) \tilde{\lambda}_{2}(t),
\end{aligned}
$$

where $\tilde{\lambda}_{3}(t) \triangleq \hat{\lambda}_{3}(t)-\lambda_{3}^{*}(t)$. Equation (28) is in a standard error model form. We propose the adaptive laws

$$
\begin{aligned}
& \dot{\hat{H}}(t)=\gamma_{H} \operatorname{Proj}\left(\hat{H}(t), \sigma\left(x_{p}(t)\right) e_{y}^{T}(t) P_{2} B\right), \\
& \dot{\hat{\lambda}}_{2}(t)=\gamma_{2} \operatorname{Proj}\left(\hat{\lambda}_{2}(t),-\operatorname{diag}\left(L_{r} \mathcal{G}(t)\right) B^{T} P_{2} e_{y}(t)\right), \\
& \dot{\hat{\lambda}}_{3}(t)=\gamma_{3} \operatorname{Proj}\left(\hat{\lambda}_{3}(t), \operatorname{diag}\left(L_{r} \Delta y(t)\right) B^{T} P_{2} e_{y}(t)\right),
\end{aligned}
$$

where $\gamma_{H}, \gamma_{2}, \gamma_{3} \in \mathbb{R}_{+}$are learning rates, and $P_{2} \in \mathbb{R}_{+}^{n \times n} \cap$ $\mathbb{S}^{n \times n}$ is the solution of $A_{m}^{T} P_{2}+P_{2} A_{m}=-Q_{2}$, for some $Q_{2} \in$ $\mathbb{R}_{+}^{n \times n} \cap \mathbb{S}^{n \times n}$. In addition, we impose bounds on the elements of $\hat{\lambda}_{2}(t)$ with each element $\hat{\lambda}_{2_{i}}(t)$ having positive upper and lower bounds $\hat{\lambda}_{2 \max _{i}}$ and $\hat{\lambda}_{2 \min _{i}}$. This ensures that $\operatorname{diag}\left(\hat{\lambda}_{2}(t)\right)$ is always invertible.
Finally, regarding the anti-windup term used in (17), and with Assumption 1 satisfied, we define $J(t)$ as

$$
J(t)=M L_{r}^{-1} \operatorname{diag}\left(\hat{\lambda}_{2}(t)\right)^{-1} L_{r},
$$

which is bounded since $\hat{\lambda}_{2 \min _{i}}>0, i=1, \ldots, m$.

Remark 2: It follows from Lemma 1 that $\tilde{\Theta}(t), \hat{\lambda}(t), \dot{\tilde{\Theta}}(t)$ and $\dot{\hat{\lambda}}(t)$ are bounded, which implies the boundedness of $H(t)$, $\Lambda_{2}(t), \dot{H}(t)$ and $\dot{\Lambda}_{2}(t)$. Therefore, there exist $h \in \mathbb{R}_{+}, \dot{h} \in \mathbb{R}_{+}$, $\beta_{3} \in \mathbb{R}_{+}$and $\dot{\beta}_{3} \in \mathbb{R}_{+}$such that $\|H(t)\| \leq h,\|\dot{H}(t)\| \leq \dot{h}$, $\left\|\Lambda_{2}(t)\right\|_{F} \leq \beta_{3}$ and $\left\|\dot{\Lambda}_{2}(t)\right\|_{F} \leq \dot{\beta}_{3}$ for all $t \geq 0$. The latter implies that $\left\|\lambda_{3}^{*}(t)\right\| \leq \beta_{3}$ and $\left\|\dot{\lambda}_{3}^{*}(t)\right\| \leq \dot{\beta}_{3}$. Moreover, as $\hat{\lambda}_{\text {min }_{i}}>0$ for $i=1, \ldots, m$, there exists $\beta_{2} \in \mathbb{R}_{+}$such that $\left\|\Lambda_{2}^{-1}(t)\right\|_{F} \leq \beta_{2}$. And since $\frac{\mathrm{d} \Lambda_{2}^{-1}}{\mathrm{~d} t}=-\Lambda_{2}^{-1} \dot{\Lambda}_{2} \Lambda_{2}^{-1}$, then there exists $\dot{\beta}_{2} \in \mathbb{R}_{+}$such that $\left\|\frac{\mathrm{d} \Lambda_{2}^{-1}}{\mathrm{~d} t}\right\|_{F} \leq \dot{\beta}_{2}$. This implies that $\left\|\lambda_{2}^{*}(t)\right\| \leq \beta_{2}$ and $\left\|\dot{\lambda}_{2}^{*}(t)\right\| \leq \dot{\beta}_{2}$ for all $t \geq 0$.

Theorem 1: Consider the uncertain dynamical system (1), the adaptive controller given by (4), (8) and (12), and the adaptive pilot model given by (17), (20), (22) and (29). Then, the solution $\left(e_{y}(t), \tilde{H}(t), \tilde{\lambda}_{2}(t), \tilde{\lambda}_{3}(t)\right)$ remains bounded for all $t \geq 0$ and converges to the compact set

$$
\begin{aligned}
& E \triangleq\left\{\left(e_{y}(t), \tilde{H}(t), \tilde{\lambda}_{2}(t), \tilde{\lambda}_{3}(t)\right):\left\|e_{y}(t)\right\|^{2} \leq \eta,\right. \\
& \left.\|\tilde{H}(t)\| \leq \tilde{h},\left\|\tilde{\lambda}_{2}(t)\right\| \leq \tilde{\alpha}_{2} \text { and }\left\|\tilde{\lambda}_{3}(t)\right\| \leq \tilde{\alpha}_{3}\right\},
\end{aligned}
$$

where

$$
\eta \triangleq \frac{2 \gamma_{H}^{-1} \tilde{h} \dot{h}+2 \gamma_{2}^{-1} \tilde{\alpha}_{2} \beta_{3} \dot{\beta}_{2}+\gamma_{2}^{-1} \tilde{\alpha}_{2} \dot{\beta}_{3} \tilde{\alpha}_{2}+2 \gamma_{3}^{-1} \tilde{\alpha}_{3} \dot{\beta}_{3}}{\lambda_{\min }\left(Q_{2}\right)},
$$

$\tilde{h} \triangleq\left\|\hat{H}_{\max }\right\|+h, \tilde{\alpha}_{2} \triangleq\left\|\hat{\lambda}_{2 \max }\right\|+\beta_{2}, \tilde{\alpha}_{3} \triangleq\left\|\hat{\lambda}_{3 \max }\right\|+\beta_{3}$, and $h, \dot{h}, \beta_{2}, \dot{\beta_{2}}, \beta_{3}$ and $\dot{\beta_{3}}$ are defined in Remark 2. Furthermore, all system signals are bounded.

Proof: Consider the Lyapunov function candidate

$$
\begin{aligned}
V_{2}\left(e_{y}, \tilde{H}, \tilde{\lambda}_{2}, \tilde{\lambda}_{3}\right)= & e_{y}^{T} P_{2} e_{y}+\gamma_{H}^{-1} \operatorname{Tr}\left\{\tilde{H}^{T} \tilde{H}\right\} \\
& +\gamma_{2}^{-1} \tilde{\lambda}_{2}^{T} \Lambda_{2} \tilde{\lambda}_{2}+\gamma_{3}^{-1} \tilde{\lambda}_{3}^{T} \tilde{\lambda}_{3} .
\end{aligned}
$$

Differentiating along the trajectories (28) and (29) yields

$$
\begin{aligned}
\dot{V}_{2}= & \dot{e}_{y}^{T} P_{2} e_{y}+e_{y}^{T} P_{2} \dot{e}_{y}+2 \gamma_{H}^{-1} \operatorname{Tr}\left\{\tilde{H}^{T} \dot{\tilde{H}}\right\} \\
& +2 \gamma_{2}^{-1} \tilde{\lambda}_{2}^{T} \Lambda_{2} \dot{\tilde{\lambda}}_{2}+\gamma_{2}^{-1} \tilde{\lambda}_{2}^{T} \dot{\Lambda}_{2} \tilde{\lambda}_{2}+2 \gamma_{3}^{-1} \tilde{\lambda}_{3}^{T} \dot{\tilde{\lambda}}_{3} \\
= & -e_{y}^{T} Q_{2} e_{y}-2 \operatorname{Tr}\left\{\tilde{H}^{T} \sigma\left(x_{p}\right) e_{y}^{T} P_{2} B\right\} \\
& +2 \tilde{\lambda}_{2}^{T} \Lambda_{2} \operatorname{diag}\left(L_{r} \mathcal{G}\right) B^{T} P_{2} e_{y}-2 \tilde{\lambda}_{3}^{T} \operatorname{diag}\left(L_{r} \Delta y\right) B^{T} P_{2} e_{y} \\
& +2 \gamma_{H}^{-1} \operatorname{Tr}\left\{\tilde{H}^{T} \hat{\hat{H}}\right\}-2 \gamma_{H}^{-1} \operatorname{Tr}\left\{\tilde{H}^{T} \dot{H}\right\} \\
& +2 \gamma_{2}^{-1} \tilde{\lambda}_{2}^{T} \Lambda_{2} \dot{\hat{\lambda}}_{2}-2 \gamma_{2}^{-1} \tilde{\lambda}_{2}^{T} \Lambda_{2} \dot{\lambda}_{2}^{*}+\gamma_{2}^{-1} \tilde{\lambda}_{2}^{T} \dot{\Lambda}_{2} \tilde{\lambda}_{2} \\
& +2 \gamma_{3}^{-1} \tilde{\lambda}_{3}^{T} \dot{\hat{\lambda}}_{3}-2 \gamma_{3}^{-1} \tilde{\lambda}_{3}^{T} \dot{\lambda}_{3}^{*} \\
= & -e_{y}^{T} Q_{2} e_{y}+\gamma_{2}^{-1} \tilde{\lambda}_{2}^{T} \dot{\Lambda}_{2} \tilde{\lambda}_{2} \\
& -2 \gamma_{H}^{-1} \operatorname{Tr}\left\{\tilde{H}^{T} \dot{H}_{3}-2 \gamma_{2}^{-1} \tilde{\lambda}_{2}^{T} \Lambda_{2} \dot{\lambda}_{2}^{*}-2 \gamma_{3}^{-1} \tilde{\lambda}_{3}^{T} \dot{\lambda}_{3}^{*}\right. \\
& +2 \operatorname{Tr}\left\{\tilde{H}^{T}\left(\operatorname{Proj}\left(\hat{H}, Y_{H}\right)-Y_{H}\right)\right\} \\
& +2 \tilde{\lambda}_{2}^{T} \Lambda_{2}\left(\operatorname{Proj}\left(\hat{\lambda}_{2}, Y_{2}\right)-Y_{2}\right) \\
& +2 \tilde{\lambda}_{3}^{T}\left(\operatorname{Proj}\left(\hat{\lambda}_{3}, Y_{3}\right)-Y_{3}\right),
\end{aligned}
$$


where $Y_{H} \triangleq \sigma\left(x_{p}\right) e_{y}^{T} P_{2} B, \quad Y_{2} \triangleq-\operatorname{diag}\left(L_{r} \mathcal{G}\right) B^{T} P_{2} e_{y}$ and $Y_{3} \triangleq \operatorname{diag}\left(L_{r} \Delta y(t)\right) B^{T} P_{2} e_{y}$. Using the projection property $\left(\theta_{i, j}-\theta_{i, j}^{*}\right)\left(\operatorname{Proj}\left(\theta_{i, j}, Y_{i, j}\right)-Y_{i, j}\right) \leq 0$, Remark 2, and the fact that $\Lambda_{2}(t)$ is diagonal positive definite, we obtain

$$
\begin{aligned}
\dot{V}_{2} \leq & -e_{y}^{T} Q_{2} e_{y}+\gamma_{2}^{-1} \tilde{\lambda}_{2}^{T} \dot{\Lambda}_{2} \tilde{\lambda}_{2} \\
& -2 \gamma_{H}^{-1} \operatorname{Tr}\left\{\tilde{H}^{T} \dot{H}\right\}-2 \gamma_{2}^{-1} \tilde{\lambda}_{2}^{T} \Lambda_{2} \dot{\lambda}_{2}^{*}-2 \gamma_{3}^{-1} \tilde{\lambda}_{3}^{T} \dot{\lambda}_{3}^{*} \\
\leq & -\lambda_{\min }\left(Q_{2}\right)\left\|e_{y}\right\|^{2}+\lambda_{\min }\left(Q_{2}\right) \eta .
\end{aligned}
$$

Hence, $\dot{V}_{2}<0$ outside the compact set (31), which proves the boundedness of $\left(e_{y}(t), \hat{H}(t), \hat{\lambda}_{2}(t), \hat{\lambda}_{3}(t)\right)$. The boundedness of all the signals in the inner-loop, including $x_{p}(t)$, follows from Lemma 1. According to (20), since $r(t)$ is bounded, $x_{m}(t)$ is also bounded, which leaves us only to prove that $x_{c}(t)$ is bounded to complete the proof.

We prove the boundedness of $x_{c}(t)$ (and hence $x(t)$ ) by considering the cases where a) $\Delta y(t)=0$ and b) $\Delta y(t) \neq 0$.

Case a) $\Delta y(t)=0$ : It follows from (27) that $e_{\Delta}(t)$ is bounded which by the fact that $e_{y}(t)$ is bounded, shows the boundedness of $e_{2}(t)$ and $x(t)$.

Case b) $\Delta y(t) \neq 0$ : Using $\Delta y(t)=y_{h}(t)-v(t),(17)$ can be rewritten as

$$
\dot{x}_{c}(t)=C_{2}^{T} x_{p}(t)-r(t)+J(t) y_{h}(t)-J(t) v(t) .
$$

Substituting (22b) and (22a), one can rewrite (36) as

$$
\begin{aligned}
\dot{x}_{c}(t)= & C_{2}^{T} x_{p}(t)-r(t)+J(t) y_{h}(t) \\
& +J(t) L_{r}^{-1} \operatorname{diag}\left(\hat{\lambda}_{2}(t)\right) L_{r} \theta_{x} x(t) \\
& +J(t) L_{r}^{-1} \operatorname{diag}\left(\hat{\lambda}_{2}(t)\right) \hat{H}^{T}(t) \sigma\left(x_{p}(t)\right) .
\end{aligned}
$$

Using (24), it is obtained by substituting $\theta_{x} x(t)=\phi x_{p}(t)+$ $\psi x_{c}(t)$ and (30) into (37) that

$$
\begin{aligned}
\dot{x}_{c}(t)= & J(t) L_{r}^{-1} \operatorname{diag}\left(\hat{\lambda}_{2}(t)\right) L_{r} \psi x_{c}(t)+w(t), \\
= & M \psi x_{c}(t)+w(t), \\
w(t) \triangleq & \left(C_{2}^{T}+M \phi\right) x_{p}(t)-r(t)+J(t) y_{h}(t) \\
& +M L_{r}^{-1} \hat{H}^{T}(t) \sigma\left(x_{p}(t)\right) .
\end{aligned}
$$

As $r(t), \hat{H}(t), y_{h}(t), J(t)$ and $x_{p}(t)$ are bounded, then so is $w(t)$. Therefore, it follows from Assumption 1 and (38) that $x_{c}(t)$ is bounded which implies the boundedness of $x(t)$.

The boundedness of $e_{2}(t)$ follows from the boundedness of $x(t)$ as $x_{m}(t)$ is bounded. Since $e_{y}(t)=e_{2}(t)-e_{\Delta}(t)$ is bounded, then $e_{\Delta}(t)$ is also bounded, which implies by (27) that $\Delta y(t)$ is bounded and completes the proof.

\section{Special Case: Systems With Free Integrators}

The assignment of $L_{r}$ given in (5) is invalid when $C_{1}^{T} A_{r}^{-1} B_{p}$

\begin{tabular}{|c|c|}
\hline$A_{n s}$ & 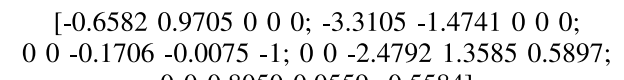 \\
\hline & 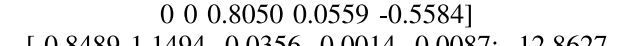 \\
\hline$A_{s}$ & 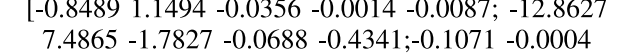 \\
\hline & $-0.6684-0.0140-1.6727 ;-0.0807-0.0474-0.6318$ \\
\hline & 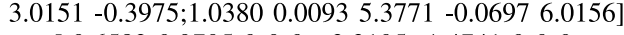 \\
\hline$A_{r s}$ & 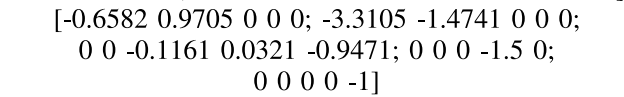 \\
\hline$B_{s}$ & $\begin{array}{c}{\left[\begin{array}{ccccccc}-0.0734 & 0 & 0 ;-3.6764 & 0 & 0 ; 0 & 0 & 0.0256 \\
0 & 1.0924 & 0.1240 ; 0 & -0.1240 & -0.2600\end{array}\right]}\end{array}$ \\
\hline & 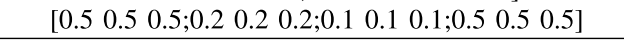 \\
\hline
\end{tabular}
is singular. This may happen, for example, when the pilot commands attitude rates to the inner-loop controller aiming to achieve desired attitude angles. In such a case, (1) can be modified by separating the states as

$$
\begin{aligned}
& {\left[\begin{array}{l}
\dot{x}_{s} \\
\dot{x}_{\theta}
\end{array}\right]=\left[\begin{array}{ll}
A_{s} & 0 \\
C_{1 s}^{T} & 0
\end{array}\right]\left[\begin{array}{l}
x_{s} \\
x_{\theta}
\end{array}\right]+\left[\begin{array}{c}
B_{s} \\
0
\end{array}\right] \Lambda\left(u_{p}(t)+W^{T} \sigma_{p}\left(x_{s}(t)\right)\right),} \\
& y_{1}(t)=\dot{x}_{\theta}(t)=C_{1 s}^{T} x_{s}(t), y_{2}(t)=x_{\theta}(t)=C_{2}^{T} x_{p}(t),
\end{aligned}
$$

where $x_{s}(t) \in \mathbb{R}^{n_{s}}, x_{\theta}(t) \in \mathbb{R}^{m}$, and $x_{p}(t) \triangleq\left[x_{s}^{T}, x_{\theta}^{T}\right]^{T} \in$ $\mathbb{R}^{n_{p}}$. To ensure the existence of $L_{r}$, the adaptive controller is
TABLE I

NUMERICAL DATA USED IN THE SIMULATIONS

made responsible only for the control of $x_{s}(t)$ dynamics while making sure that the human input $y_{h}(t)$ is being tracked by the plant. This yields inner-loop dynamics similar to (10) as

$$
\begin{aligned}
\dot{x}_{s}(t)= & A_{r s} x_{s}(t)+B_{r s} y_{h}(t)+B_{s} \Lambda \operatorname{diag}(\tilde{\lambda}(t)) L_{r} y_{h}(t) \\
& -B_{S} \Lambda \tilde{\Theta}^{T}(t) \sigma\left(x_{s}(t)\right),
\end{aligned}
$$

where the pair $\left(A_{r s}, B_{r s}\right)$ describe the modified reference model dynamics with the nominal dynamics of $x_{S}(t)$ represented by $\left(A_{n s}, B_{s}\right)$. Augmenting (40) with $x_{\theta}(t)$ dynamics results in a closed loop system similar to (16) which directly extends our previous results to the case in hand.

\section{Simulations}

Consider a large transport aircraft model [14] linearized at Mach 3 and altitude $1000 \mathrm{~m}$ in the form of (39), where

$$
x_{s}(t)=\left[\begin{array}{lllll}
\alpha & q & \beta & p & r
\end{array}\right]^{T}, \dot{x}_{\theta}(t)=\left[\begin{array}{lll}
p & q & r
\end{array}\right]^{T} \text {, }
$$

$\alpha$ (rad) is the angle of attack, $\beta$ (rad) is the side-slip angle, and $p, q$ and $r(\mathrm{rad} / \mathrm{s})$ are the roll, pitch and yaw rates, respectively. The input vector is defined as

$$
u_{p}(t)=\left[\begin{array}{lll}
\delta_{e} & \delta_{a} & \delta_{r}
\end{array}\right]^{T},
$$

consisting of the deflections (in rad) of 2 ganged elevators $\delta_{e}$, 2 ganged ailerons $\delta_{a}$ and 2 ganged rudders $\delta_{r}$. The numerical data used in the simulations are given in Table I.

The matched system uncertainty $W_{p}^{T} \sigma_{p}\left(x_{s}(t)\right)$ is given by $\sigma_{p}\left(x_{s}(t)\right) \triangleq[1, q, p, r]^{T}$. A pilot is controlling the aircraft to achieve desired attitude angles by feeding attitude rate commands to the inner-loop controller using a side-stick.

To design the crossover-reference model (20), the LQR method is used with $Q_{L Q R}=\operatorname{diag}\left(\left[0_{1 \times 8}, 5,5,5\right]\right)$ and $R_{L Q R}=$ $5 I_{3 \times 3}$ which yields $\theta_{x}$ in (24) with the sub-matrix $\psi=I_{3 \times 3}$. As all eigen values of $\psi$ have positive real parts, we assume $M=-100 I_{3 \times 3}$ which ensures that $M \psi$ is Hurwitz. In addition, the Lyapunov matrices are taken as $Q_{1}=I_{5 \times 5}$ and $Q_{2}=2 I_{11 \times 11}$, and the human pilot's learning rates are assumed to be $\gamma_{H}=\gamma_{2}=\gamma_{3}=20$. The human's command is saturated as in (22c) with $y_{o_{i}}=20 \mathrm{deg} / \mathrm{s}$ for $i=1, \ldots, 3$, $\Lambda=I_{3 \times 3}$ for $t<40 \mathrm{sec}$, and we introduce a failure into the system by making $\Lambda=0.3 I_{3 \times 3}$ for $t \geq 40 \mathrm{sec}$.

Figs. 2 and 3 show the aircraft attitude angles and the evolution of the pilot commands, respectively for different innerloop learning rates. Good tracking performance is observed 

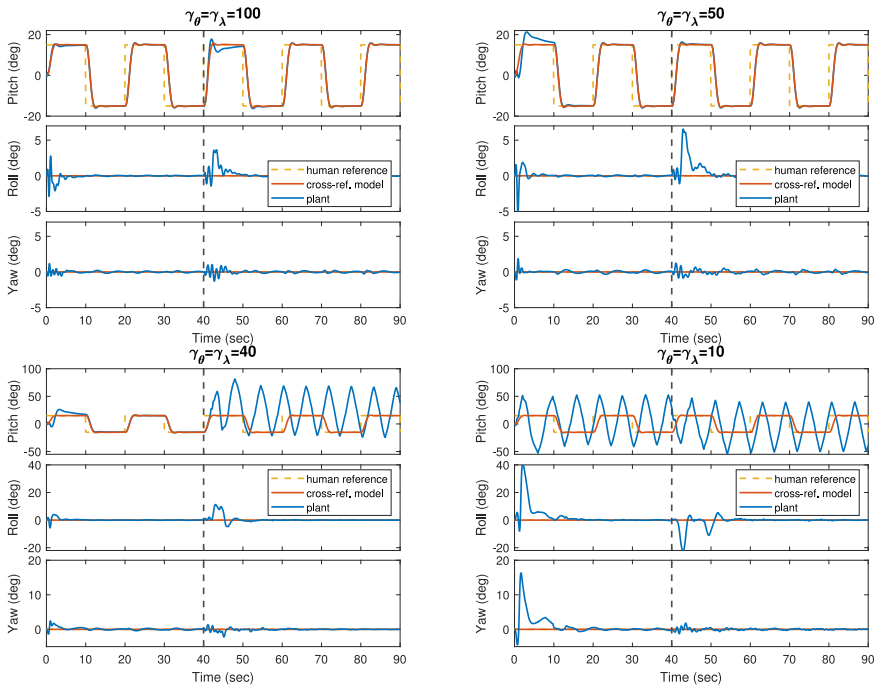

Fig. 2. Attitude angles for different inner-loop learning rates.
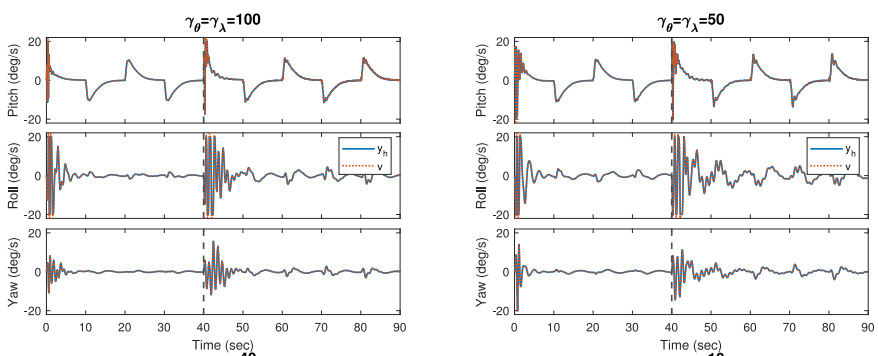

$\gamma_{\theta}=\gamma_{\lambda}=4$
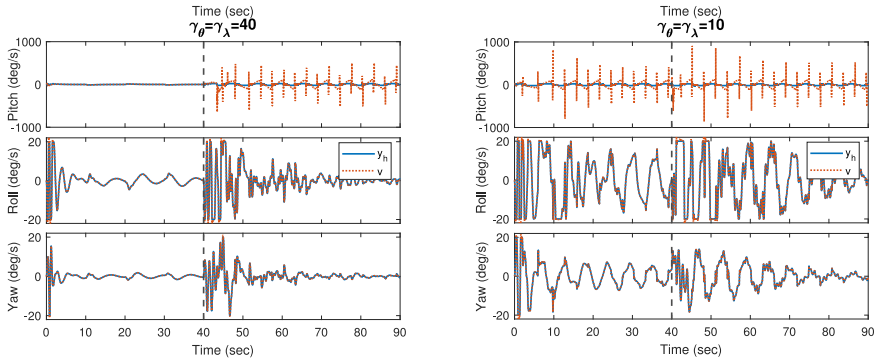

Fig. 3. Pilot commands for different inner-loop learning rates.

for the cases where $\gamma_{\theta}=\gamma_{\lambda}=100$ and $\gamma_{\theta}=\gamma_{\lambda}=50$ while the pilot commands show an oscillatory behavior when an uncertainty is introduced to the system. However, for the cases where $\gamma_{\theta}=\gamma_{\lambda}=40$ and $\gamma_{\theta}=\gamma_{\lambda}=10$, despite the boundedness of all the signals, the tracking performance significantly deteriorates resulting in consistent high amplitude oscillations. Furthermore, pilot commands saturate in the pitch direction since the pilot spends more effort to maintain a satisfactory performance due to a slower adaptive controller.

The simulations in Figs. 2 and 3 are not aimed towards providing high-performance controller implementation examples, but rather to demonstrate a use case for the presented modeling approach where the effect of the adaptive controller learning rate on the behavior of the pilot is investigated.

\section{REFERENCES}

[1] D. McRuer and D. Graham, "Pilot-vehicle control system analysis," in Proc. Guid. Control Conf., 1963, p. 310.

[2] M. Lone and A. Cooke, "Review of pilot models used in aircraft flight dynamics," Aerosp. Sci. Technol., vol. 34, pp. 55-74, Apr. 2014.

[3] S. Xu, W. Tan, A. V. Efremov, L. Sun, and X. Qu, "Review of control models for human pilot behavior," Annu. Rev. Control, vol. 44 pp. 274-291, Oct. 2017.

[4] R. A. Hess, "Modeling pilot control behavior with sudden changes in vehicle dynamics," J. Aircraft, vol. 46, no. 5, pp. 1584-1592, 2009.

[5] R. A. Hess, "Modeling human pilot adaptation to flight control anomalies and changing task demands," J. Guid. Control Dyn., vol. 39, no. 3 , pp. 655-666, 2016.

[6] S. S. Tohidi and Y. Yildiz, "A control theoretical adaptive human pilot model: Theory and experimental validation," 2020, arXiv:2007.10216.

[7] S. S. Tohidi and Y. Yildiz, "Adaptive human pilot model for uncertain systems," in Proc. 18th Eur. Control Conf. (ECC), 2019, pp. 2938-2943.

[8] T. Yucelen, Y. Yildiz, R. Sipahi, E. Yousefi, and N. Nguyen, "Stability limit of human-in-the-loop model reference adaptive control architectures," Int. J. Control, vol. 91, no. 10, pp. 2314-2331, 2018.

[9] S. Tohidi and Y. Yildiz, "Adaptive control allocation: A humanin-the-loop stability analysis," IFAC-PapersOnLine, vol. 53, no. 2, pp. 6321-6326, 2020

[10] D. Klyde, C.-Y. Liang, D. Alvarez, N. Richards, R. Adams, and B. Cogan, "Mitigating unfavorable pilot interactions with adaptive controllers in the presence of failures/damage," in Proc. AIAA Atmos. Flight Mech. Conf., 2011, p. 6538.

[11] M. Steinberg, "Historical overview of research in reconfigurable flight control," Proc. Inst. Mech. Eng. G, J. Aerosp. Eng., vol. 219, no. 4, pp. 263-275, 2005.

[12] E. Lavretsky and K. A. Wise, "Robust adaptive control," in Robust and Adaptive Control. London, U.K.: Springer, 2013, pp. 317-353.

[13] M. Schwager and A. M. Annaswamy, "Direct adaptive control of multiinput plants with magnitude saturation constraints," in Proc. 44th IEEE Conf. Decis. Control, 2005, pp. 783-788.

[14] M. Schwager, A. M. Annaswamy, and E. Lavretsky, "Adaptation-based reconfiguration in the presence of actuator failures and saturation," in Proc. Amer. Control Conf., 2005, pp. 2640-2645.

[15] N. Moustakis, S. Yuan, and S. Baldi, "An adaptive design for quantized feedback control of uncertain switched linear systems," Int. J. Adapt. Control Signal Process., vol. 32, no. 5, pp. 665-680, 2018.

[16] S. S. Tohidi, Y. Yildiz, and I. V. Kolmanovsky, "Adaptive control allocation for constrained systems," Automatica, vol. 121, Jun. 2020, Art. no. 109161.

[17] S. S. Tohidi, Y. Yildiz, and I. V. Kolmanovsky, "Pilot induced oscillation mitigation for unmanned aircraft systems: An adaptive control allocation approach," in Proc. IEEE Conf. Control Technol. Appl. (CCTA), 2018, pp. 343-348.

[18] J. A. Boudreau and H. L. Berman, "Dispersed and reconfigurable digital flight control system," Grumman Aerosp. Corporat., Falls Church, VA USA, 1979.

[19] K. Graham, T. B. Cunninham, and C. Shure, "Aircraft flight control survivability through use of computational techniques," Naval Air Develop. Center, Tech. Rep. 77028-30, 1980.

[20] A. Habboush and Y. Yildiz, "Modeling pilot-flight control system interactions in the presence of uncertainty," in Proc. AIAA SciTech, 2022.

[21] K. J. Astrom and L. Rundqwist, "Integrator windup and how to avoid it," in Proc. Amer. Control Conf., 1989, pp. 1693-1698.

[22] N. Kapoor, A. R. Teel, and P. Daoutidis, "An anti-windup design for linear systems with input saturation," Automatica, vol. 34, no. 5, pp. 559-574, 1998 .

[23] S. P. Karason and A. M. Annaswamy, "Adaptive control in the presence of input constraints," in Proc. Amer. Control Conf., 1993 , pp. 1370-1374. 